\title{
Índices e espacialização da erosividade das chuvas para o Estado do Rio de Janeiro ${ }^{1}$
}

\author{
Flávio A. Gonçalves ${ }^{2}$, Demetrius D. da Silva ${ }^{2}$, Fernando F. Pruski ${ }^{2}$, Daniel F. de Carvalho ${ }^{3}$ \& Eleandro S. da Cruz $^{3}$
}

\begin{abstract}
RESUMO
Visando aprimorar o conhecimento sobre a erosividade das chuvas no Estado do Rio de Janeiro objetivou-se, no presente trabalho, calcular os índices de erosividade $\mathrm{El}_{30}$ e $\mathrm{KE}>25$, utilizando-se duas metodologias para estimativa da energia cinética, estimar os índices de erosividade mensais médios mediante os dados pluviométricos e obter os mapas de isoerosividade para o referido Estado. Com base nos dados pluviográficos de 14 estações localizadas no Rio de Janeiro, obteve-se o $\mathrm{El}_{30}$ e o $\mathrm{KE}>25$. A estimativa dos índices também foi feita por meio de correlações com a precipitação mensal média ou com o coeficiente de chuva e a espacialização, por meio de mapas gerados com o uso do software ArcView 3.2a. Os resultados permitiram concluir-se que os índices de erosividade apresentam, para os diferentes locais estudados, diferenças expressivas entre si; não houve diferenças significativas entre os resultados obtidos pelas equações propostas para o cálculo da energia cinética da chuva, e a estimativa da erosividade das chuvas a partir de precipitação mensal média, proporcionou melhores resultados que quando se usou coeficiente de chuva.
\end{abstract}

Palavras-chave: perdas de solo, erosão hídrica, dados pluviográficos

\section{Indices and spatialization of rainfall erosivity in Rio de Janeiro State, Brazil}

\begin{abstract}
For improving the knowledge about rainfall erosivity in the state of Rio de Janeiro, Brazil, the present study aimed to calculate the erosivity indices $\mathrm{El}_{30}$ and $\mathrm{KE}>25$ using two methodologies to estimate the kinetic energy, average monthly erosivity indices based on pluviometric data and to obtain the isoerosivity maps for the State. $\mathrm{El}_{30}$ and $\mathrm{KE}>25$ were obtained using rain gauge data of 14 stations located in Rio de Janeiro. Index estimates were also obtained by using correlations with the average monthly precipitation or the rainfall coefficient and spatialization via maps generated by ArcView 3.2a software. The results permit to conclude that the erosivity indices, for the different studied places, present expressive differences among themselves; there were no significant differences between the results obtained by the equations proposed for the calculation of rain kinetic energy and the estimate of rainfall erosivity using the average monthly precipitation gave better results compared to based on rainfall coefficient.
\end{abstract}

Key words: soil losses, water erosion, rain gauge data

1 Parte da Dissertação de Mestrado do primeiro autor. Projeto financiado parcialmente com recursos do CNPq.

2 DEA/UFV. Av. P.H. Rolfs s/n CEP 36571-00 Viçosa, MG. Fone: (31) 3899-1925. E-mail: flaviogoncalves@vicosa.ufv.br; david@ufv.br (Fone: 3899-1904); ffpruski@ufv.br (Fone: 3899-1912)

${ }_{3}^{3}$ Departamento de Engenharia/UFRRJ. BR 465, km 7, CEP:23890-000, Seropédica, RJ. Fone: (21) 2682 1865. E-mail: carvalho@ufrrj.br; el-cruz@bol.com.br 


\section{INTRODUÇÃO}

A necessidade de se obter uma metodologia capaz de avaliar os fatores que causam a erosão hídrica e de estimar as perdas de solo, resultou no desenvolvimento da Equação Universal de Perdas de Solo (USLE), que permite a previsão dessas perdas e a identificação dos fatores que exercem o maior efeito sobre elas. Entre os componentes da USLE, o que expressa a capacidade erosiva da chuva é conhecido como erosividade das chuvas e caracterizado como fator $\mathrm{R}$ (Wischmeier \& Smith, 1958).

Vários são os parâmetros que podem ser utilizados para estimar a erosividade da chuva; o problema é escolher o mais adequado, uma vez que cada ambiente e evento são únicos nas escalas temporal e espacial e, conseqüentemente, a erosão varia de diferentes maneiras. Nas regiões de clima temperado, por exemplo, a variável que tem fornecido melhores resultados para avaliar a erosividade da chuva é o produto da sua energia cinética (EC) por sua intensidade máxima em 30 min $\left(\mathrm{I}_{30}\right)$, sendo expressa como $\mathrm{EI}_{30}$, porém alguns autores (Hudson, 1973; Lal, 1988) verificaram que o $\mathrm{EI}_{30}$ não apresenta boa correlação com as perdas de solo em regiões tropicais, propondo uma metodologia alternativa para essas regiões, chamada $\mathrm{KE}>25$, que é a soma da energia cinética dos segmentos da chuva com intensidade superior a $25 \mathrm{~mm} \mathrm{~h}^{-1}$.

$\mathrm{Na}$ determinação dos índices de erosividade $\mathrm{EI}_{30} \mathrm{e}$ $\mathrm{KE}>25$, é fundamental a existência de uma série histórica de dados pluviográficos. Quando em certa região esses dados são escassos, a estimativa do índice de erosividade pode ser realizada com base em dados pluviométricos, que são mais fáceis de serem obtidos que os dados pluviográficos.

Em várias regiões do Brasil os índices de erosividade vêm sendo utilizados no traçado de mapas de linhas isoerosivas. A obtenção dessas linhas é uma alternativa para contornar a escassez de dados que possibilitem a determinação dos índices de erosividade em localidades que carecem de tais informações. Esses estudos, entretanto, ainda não haviam sido realizados para o Estado do Rio de Janeiro, que possui extensas áreas com potencial agrícola localizadas, muitas vezes, em regiões com topografia acidentada e altos índices pluviométricos.
Sabendo do significado que a erosividade da chuva possui no processo erosivo objetivou-se, neste trabalho, calcular os índices de erosividade das chuvas $\mathrm{EI}_{30}$ e $\mathrm{KE}>25$ para 14 localidades do Estado do Rio de Janeiro, com disponibilidade de dados pluviográficos, utilizando-se duas metodologias para a estimativa da energia cinética; estimar os índices de erosividade mensais médios nas referidas localidades com base em dados pluviométricos e obter os mapas de isoerosividade para este Estado.

\section{MATERIAL E MÉTODOS}

Este estudo foi realizado com dados de precipitação referentes às estações pluviográficas localizadas no Estado do Rio de Janeiro e pertencentes às redes hidrometeorológicas da ANA, Light e SERLA. Utilizaram-se os registros de pluviogramas diários, com amplitude de $10 \mathrm{~mm}$ de altura precipitada e menor escala de leitura de $0,1 \mathrm{~mm}$ e tempo de registro de $24 \mathrm{~h}$ com menor escala de leitura de $10 \mathrm{~min}$.

Na Tabela 1 encontra-se a listagem com o código e o nome das 14 estações pluviográficas utilizadas neste estudo, a entidade a que pertencem, a localização, a altitude e o período de anos de observação de cada estação analisada. Devido às falhas nas séries históricas, não foi possível selecionar um período comum de dados para todas as estações pluviográficas, denominado período-base, para fins de determinação dos índices de erosividade das chuvas. Na Figura 1 tem-se o mapa do Estado do Rio de Janeiro com as estações pluviográficas empregadas no estudo.

De acordo com Wischmeier \& Smith (1978), para se obter um fator R confiável, estima-se que sejam necessários de 10 a 20 anos de coleta de dados, porém outros trabalhos sobre erosividade têm sido realizados, abrangendo um número de anos inferior ao recomendado, em função da grande dificuldade de obtenção da base de dados pluviográficos (Rufino, 1986; Medina \& Oliveira Júnior, 1987; Moura \& Medeiros, 1987; Morais et al., 1991; Marques et al., 1997). No presente trabalho, 11 das 14 estações pluviográficas utilizadas apresentavam de 14 a 19 anos de dados; entretanto, as Estações Manuel Duarte, Vila Mambucaba e Santa Isabel do

Tabela 1. Caracterização das estações pluviográficas situadas no Estado do Rio de Janeiro e utilizadas no estudo

\begin{tabular}{|c|c|c|c|c|c|c|c|}
\hline Estação & Entidade & Município & Latitude $\left({ }^{0}\right)$ & Longitude $\left({ }^{\circ}\right)$ & Altitude (m) & Período & Anos Observados \\
\hline Manuel Duarte (MD) & ANA & Rio das Flores & $-22,08$ & $-43,56$ & 400 & 1989-1992 & 5 \\
\hline Santa Isabel do Rio Preto (SI) & ANA & Valença & $-22,23$ & $-44,06$ & 567 & $1990-1996$ & 7 \\
\hline Vila Mambucaba (VM) & ANA & Angra dos Reis & $-23,03$ & $-44,52$ & 6 & $1991-1996$ & 6 \\
\hline Ilha dos Pombos (IP) & Light & Carmo & $-21,84$ & $-42,58$ & 146 & 1976-1991 & 15 \\
\hline Santa Cecília (SC) & Light & Barra do Piraí & $-22,48$ & $-43,84$ & 371 & $1963-1996$ & 14 \\
\hline Lajes (L) & Light & Piraí & $-22,70$ & $-43,88$ & 462 & $1966-1983$ & 15 \\
\hline Tocos(T0) & Light & Rio Claro & $-22,75$ & $-44,12$ & 479 & $1981-1999$ & 15 \\
\hline Tanguá (TA) & SERLA & Rio Bonito & $-22,71$ & $-42,70$ & 40 & $1980-1995$ & 16 \\
\hline Escola União (EU) & SERLA & Magé & $-22,58$ & $-42,94$ & 10 & 1977-1995 & 19 \\
\hline Cachoeiras de Macacu (CM) & SERLA & C. de Macacu & $-22,48$ & $-42,66$ & 40 & $1979-1993$ & 15 \\
\hline Posto Garrafão (PG) & SERLA & Magé & $-22,48$ & $-43,00$ & 640 & $1980-1995$ & 16 \\
\hline Rio Mole (RM) & SERLA & Saquarema & $-22,85$ & $-42,55$ & 10 & $1980-1994$ & 15 \\
\hline Eletrobrás (E) & SERLA & R. de Janeiro & $-22,92$ & $-43,42$ & 40 & 1979-1995 & 17 \\
\hline Capela Mayrink (CMA) & SERLA & R. de Janeiro & $-22,96$ & $-43,28$ & 460 & 1980-1995 & 16 \\
\hline
\end{tabular}




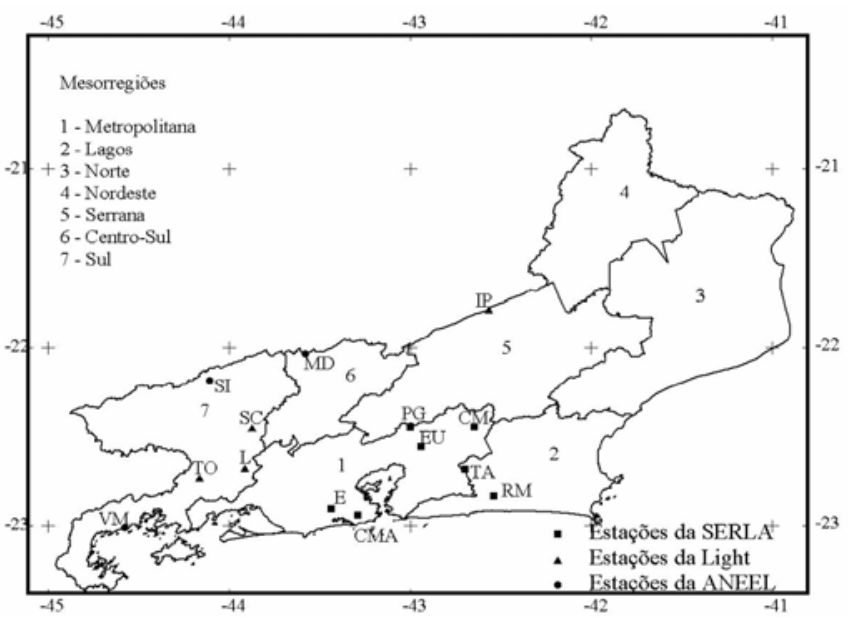

Figura 1. Mapa do Estado do Rio de Janeiro subdividido em mesorregiões, com as estações pluviográficas da ANA, Light e SERLA

Rio Preto, pertencentes à rede hidrometeorológica da ANA, apresentavam apenas de cinco a sete anos de observações. A utilização dessas estações, mesmo em se considerando o pequeno período de observações, deveu-se ao fato de se localizarem em regiões do Estado do Rio de Janeiro que não apresentavam outras informações disponíveis e, por isso, sua exclusão do estudo prejudicaria a obtenção dos mapas de isoerosividades.

Os dados pluviográficos foram convertidos para o formato digital através da digitalização com uso do Sistema para Digitalização de Pluviogramas (HidroGraph 1.02), desenvolvido pelo Grupo de Pesquisa em Recursos Hídricos (http:// www.ufv.br/dea/gprh) do Departamento de Engenharia Agrícola da Universidade Federal de Viçosa.

Os critérios adotados para individualização das chuvas erosivas foram baseados naqueles propostos por Wischmeier \& Smith (1965) e modificados por Cabeda (1976). De posse dos pluviogramas, consideraram-se chuvas erosivas aquelas que apresentaram altura total precipitada igual ou superior a $10 \mathrm{~mm}$. Para totais precipitados inferiores a $10 \mathrm{~mm}$, as chuvas foram consideradas erosivas apenas quando a lâmina precipitada em $15 \mathrm{~min}$ foi de $6 \mathrm{~mm}$ ou mais.

Eventos independentes foram considerados como os ocorridos em intervalo igual ou superior a seis horas consecutivas em que a precipitação, nesse intervalo, foi inferior a $1 \mathrm{~mm}$.

A energia cinética das chuvas foi determinada por meio das equações sugeridas por Foster et al. (1981) que converte os dados para o Sistema Internacional de Unidades (Eq. 1), e pela metodologia proposta por Wagner \& Massambani (1988) que permite relacionar a energia cinética e a taxa de precipitação de chuvas predominantemente convectivas (Eq. 2):

$$
\begin{aligned}
& \mathrm{EC}=0,119+0,0873 \log \mathrm{I} \\
& \mathrm{EC}=0,153+0,0645 \log \mathrm{I}
\end{aligned}
$$

em que:

EC - energia cinética, em $\mathrm{MJ} \mathrm{ha}^{-1} \mathrm{~mm}^{-1}$

I - intensidade de chuva, em $\mathrm{mm} \mathrm{h}^{-1}$
Segundo Foster et al. (1981), o diâmetro das gotas de chuva não aumenta quando as intensidades são iguais ou superiores a $76 \mathrm{~mm} \mathrm{~h}^{-1}$ e, desta forma, a energia cinética passa a ter um valor máximo de $0,283 \mathrm{MJ} \mathrm{ha}^{-1} \mathrm{~mm}^{-1}$.

$\mathrm{Na}$ determinação dos índices de erosividade $\mathrm{EI}_{30} \mathrm{e}$ $\mathrm{KE}>25$ fez-se uso de tabelas fornecidas pelo software HidroGraph 1.02, com os valores precipitados interpolados, em intervalos de $10 \mathrm{~min}$, que foram importados para planilhas eletrônicas do Microsoft Excel, onde os dados foram manipulados; posteriormente, fez-se a determinação da intensidade para cada um dos intervalos, em $\mathrm{mm} \mathrm{h}^{-1}$, com base na tabela com os valores precipitados em intervalos de $10 \mathrm{~min}$. Foram determinadas as energias cinéticas parciais para cada uma das intensidades, nos respectivos intervalos, com o uso das equações propostas por Foster et al. (1981) e Wagner \& Massambani (1988).

Para a determinação do índice de erosividade $\mathrm{EI}_{30}$ fezse a soma dos valores do produto das energias cinéticas parciais calculadas para a intensidade de cada intervalo, em MJ ha-1 $\mathrm{mm}^{-1}$, pela altura precipitada no respectivo intervalo, em mm, resultando na energia total da chuva, em MJ ha-1 . Obteve-se a máxima intensidade de precipitação em 30 min consecutivos $\left(\mathrm{I}_{30}\right)$ e, por último, o índice $\mathrm{EI}_{30}$, em MJ mm ha-1 $\mathrm{h}^{-1}$, pela multiplicação da energia cinética total pela intensidade de precipitação máxima em 30 min consecutivos.

Para a determinação do índice de erosividade KE > 25 fez-se a determinação das energias cinéticas parciais para as intensidades superiores ou iguais a $25 \mathrm{~mm} \mathrm{~h}^{-1}$, nos respectivos intervalos, com uso das equações sugeridas por Foster et al. (1981) e Wagner \& Massambani (1988). Somaram-se os valores do produto das energias cinéticas parciais, calculadas para intensidades maiores que $25 \mathrm{~mm} \mathrm{~h}^{-1}$, em MJ ha-1 $\mathrm{mm}^{-1}$, pela altura precipitada nos respectivos intervalos, em mm, resultando na energia total da chuva, em MJ ha-1.

Pelo somatório dos valores dos índices $\mathrm{EI}_{30}$ e $\mathrm{KE}>25$ das chuvas erosivas ocorridas em cada mês, obtiveram-se os índices $\mathrm{EI}_{30}$ e $\mathrm{KE}>25$ mensais. O fator de erosividade da chuva (R) foi conseguido pela soma dos valores das médias mensais da série histórica desses índices, caso em que se obteve um fator $\mathrm{R}$ relativo ao índice $\mathrm{EI}_{30}$ e outro relativo ao $\mathrm{KE}>25$, para cada uma das metodologias de obtenção da energia cinética. Como foram duas metodologias, isto resultou em quatro índices de erosividade.

A estimativa dos índices de erosividade mensais médios a partir de dados pluviométricos, foi feita por meio de correlações dos índices de erosividade, obtidos a partir da base de dados pluviográficos, com a precipitação mensal média e com o coeficiente de chuva (Rc). Este coeficiente foi obtido empregando-se os dados pluviométricos mensais médios correspondentes a cada uma das estações utilizadas no estudo de erosividade, com base na Eq.3, conforme apresentada por Silva et al. (1997) e proposta por Lombardi Neto (1977):

$$
\mathrm{Rc}=\frac{\mathrm{p}^{2}}{\mathrm{P}}
$$


em que:

$$
\begin{aligned}
\text { Rc - coeficiente de chuva, mm } \\
\text { p - precipitação mensal média, mm } \\
\text { P - precipitação anual média, mm }
\end{aligned}
$$

Ajustou-se uma equação de regressão que correlacionou o índice de erosividade $\mathrm{EI}_{30}$ mensal médio, obtido a partir dos dados pluviográficos, com a precipitação mensal média e outra equação, com o coeficiente de chuva (Rc). Para o índice KE > 25 mensal médio, procedeu-se da mesma forma, ajustando-se outras duas equações de regressão com a precipitação mensal média e com o coeficiente de chuva.

O objetivo dos ajustes das equações de regressão foi escolher aquelas que melhor estimassem os índices de erosividades $\mathrm{EI}_{30}$ e $\mathrm{KE}>25$ mensais médios, originados de dados pluviométricos, para cada uma das 14 localidades analisadas. As equações obtidas podem ser utilizadas para a estimativa da erosividade da chuva em outras localidades que não disponham de informações pluviográficas mas que tenham características de chuvas semelhantes às dos locais estudados.

Desta forma, obtiveram-se oito equações de regressão para cada uma das localidades, já que se tratava de duas metodologias para o cálculo da energia cinética das chuvas e de dois índices de erosividade da chuva, obtidos a partir de dados pluviográficos e pluviométricos (precipitação mensal média e coeficiente de chuva).

A metodologia utilizada na elaboração dos mapas temáticos com os índices de erosividade $\mathrm{EI}_{30}$ e $\mathrm{KE}>25$ para o Estado do Rio de Janeiro constou, basicamente, dos seguintes procedimentos:

- do uso do software de sistemas de informações geográficas ArcView GIS 3.2a com uma base de dados contendo o contorno do Estado do Rio de Janeiro em formato "shape" que foi, posteriormente, convertido em formato "grid" e uma tabela de atributos em formato "dbf" contendo, além das coordenadas geográficas, os índices de erosividade anuais médios $\mathrm{EI}_{30}$ e $\mathrm{KE}>25$ de cada estação analisada;

- da obtenção dos mapas "erosividade $\mathrm{EI}_{30}$ " contendo 12 classes de erosividade, com variação de $1.000 \mathrm{MJ} \mathrm{mm} \mathrm{ha}^{-1} \mathrm{~h}^{-1} \mathrm{ano}^{-1} \mathrm{e}$ "erosividade KE > 25", com 14 classes e variação de $10 \mathrm{MJ} \mathrm{ha}^{-1} \mathrm{ano}^{-1}$.

\section{RESULTADOS E DISCUSSÃO}

Na Tabela 2 encontram-se os valores anuais médios dos índices de erosividade $\mathrm{EI}_{30}$ e $\mathrm{KE}>25$ das 14 estações do Estado do Rio de Janeiro. Os índices de erosividade, calculados por meio da equação de energia cinética sugerida por Foster et al. (1981) receberam o índice “ 1 ” e os calculados por intermédio da equação proposta por Wagner \& Massambani (1988) o índice “2”.

Pode-se notar, nos dados apresentados nesta tabela, que existem apenas pequenas diferenças nos resultados de erosividade quando se analisam as duas equações de energia cinética empregadas nos cálculos; resultados semelhantes foram verificados por McGregor et al. (1995) e Marques et al. (1997).

\begin{tabular}{|c|c|c|c|c|}
\hline \multirow{3}{*}{ Estação } & \multicolumn{4}{|c|}{ Índices de Erosividade } \\
\hline & $\left(\mathrm{El}_{30}\right)_{1}$ & $\left(\mathrm{El}_{30}\right)_{2}$ & $(\mathrm{KE}>25)_{1}$ & $(\mathrm{KE}>25)_{2}$ \\
\hline & \multicolumn{2}{|c|}{ MJ mm ha-1 $\mathrm{h}^{-1}$ ano $^{-1}$} & \multicolumn{2}{|c|}{ MJ ha-1 ano-1 } \\
\hline Cachoeiras de Macacu & 7.961 & 8.233 & 94,2 & 94,0 \\
\hline Capela Mayrink & 9.331 & 9.705 & 81,0 & 80,2 \\
\hline Eletrobrás & 4.439 & 4.626 & 51,1 & 50,6 \\
\hline Escola União & 10.235 & 10.502 & 119,3 & 118,1 \\
\hline Ilha dos Pombos & 5.653 & 5.819 & 72,5 & 71,8 \\
\hline Lajes & 6.696 & 6.908 & 80,2 & 79,4 \\
\hline Manuel Duarte & 4.118 & 4.268 & 42,1 & 41,7 \\
\hline Posto Garrafão & 15.806 & 16.225 & 171,8 & 170,2 \\
\hline Rio Mole & 5.448 & 5.626 & 61,6 & 61,0 \\
\hline Santa Cecília & 4.985 & 5.140 & 62,3 & 61,8 \\
\hline Santa I. do R. Preto & 6.971 & 7.188 & 88,6 & 87,9 \\
\hline Tanguá & 5.289 & 5.469 & 62,2 & 61,5 \\
\hline Tocos & 9.031 & 9.267 & 115,8 & 114,8 \\
\hline Vila Mambucaba & 10.140 & 10.485 & 90,9 & 90,3 \\
\hline
\end{tabular}

Tabela 2. Valores anuais médios dos índices de erosividade $\mathrm{El}_{30} \mathrm{e}$ KE $>25$ das diferentes localidades do Estado do Rio de Janeiro

$\mathrm{O}$ índice de erosividade anual médio $\mathrm{EI}_{30}$ variou de 4.118 MJ mm ha-1 $\mathrm{h}^{-1}$ ano $^{-1}$ na Estação Manuel Duarte, a 16.225 MJ mm ha-1 $\mathrm{h}^{-1}$ ano $^{-1}$ na Estação Posto Garrafão. O valor do $\mathrm{EI}_{30}$ anual médio de Manuel Duarte foi semelhante ao de Pelotas, RS, (3.924 MJ mm ha-1 $\mathrm{h}^{-1}$ ano $^{-1}$ ), obtido por Lago (1984), e das localidades paraibanas de Areia, Itaporama e Guarabira (3.875, 4.150 e $4.159 \mathrm{MJ} \mathrm{mm} \mathrm{ha}^{-1} \mathrm{~h}^{-1} \mathrm{ano}^{-1}$, respectivamente) determinados por Chaves \& Diniz (1981).

Os valores apresentados na literatura que mais se aproximam do índice de erosividade anual médio obtido em Posto Garrafão, são os de Manaus, AM, (14.129 MJ mm ha $\left.{ }^{-1} \mathrm{~h}^{-1} \mathrm{ano}^{-1}\right)$, conseguidos por Oliveira Júnior \& Medina (1990), e dos municípios paraenses de Bragança e Marabá (12.350 e 13.914 MJ mm ha-1 $\mathrm{h}^{-1} \mathrm{ano}^{-1}$, respectivamente), obtidos por Oliveira Júnior (1988). Na Nigéria, Salako et al. (1995) encontraram valor de $\mathrm{EI}_{30}$ de $18.510 \mathrm{MJ} \mathrm{mm} \mathrm{ha}^{-1} \mathrm{~h}^{-1}$ ano $^{-1}$. O índice KE > 25 anual médio variou de 41,7 $\mathrm{MJ}^{-1} \mathrm{ha}^{-1}$ ano $^{-1}$ na Estação Manuel Duarte a $171,8 \mathrm{MJ}_{\text {ha }}{ }^{-1} \mathrm{ano}^{-1}$ na Estação Posto Garrafão.

Fazendo-se a comparação entre os índices de erosividade calculados por meio da equação de energia cinética sugerida por Foster et al. (1981) e daquela proposta por Wagner \& Massambani (1988), observa-se que, para o índice $\mathrm{EI}_{30}$, os valores encontrados utilizando-se a equação de energia cinética recomendada por aqueles autores foram, em média, 3,15\% menores que os obtidos através da equação de energia cinética proposta por estes últimos. Para o índice $\mathrm{KE}>25$, ocorreu inversão, ou seja, os valores encontrados através da equação de energia cinética sugerida por Foster et al. (1981) passaram a ser, em média, 0,88\% maiores que os obtidos utilizando-se a equação de energia cinética proposta por Wagner \& Massambani (1988). A explicação para isto foi que a equação sugerida por aqueles pesquisadores forneceu valores maiores de energia cinética que a proposta por esses dois, quando a intensidade da chuva foi superior a $31 \mathrm{~mm} \mathrm{~h}^{-1}$; por este motivo, os valores do índice KE $>25$, 
que levam em conta as intensidades das chuvas superiores a $25 \mathrm{~mm} \mathrm{~h}^{-1}$ e foram obtidos por meio da equação sugerida por Foster et al. (1981), mostraram-se sempre superiores ou iguais aos do índice $\mathrm{KE}>25$ obtidos por intermédio da equação proposta por Wagner \& Massambani (1988); o mesmo não ocorre com o $\mathrm{EI}_{30}$ e, por esta razão, os valores obtidos por intermédio da equação de energia cinética sugerida por Foster et al. (1981) foram menores que os encontrados utilizando-se a equação de energia cinética proposta por Wagner \& Massambani (1988). Esta constatação também foi verificada por Marques et al. (1988).

Em virtude da pequena variação percentual média entre os valores dos índices de erosividade $\mathrm{EI}_{30}$ e $\mathrm{KE}>25$ (3,15 e $0,88 \%$, respectivamente), obtidos pelas diferentes equações para o cálculo da energia cinética, somente serão discutidos os resultados alcançados com base na equação sugerida por Foster et al. (1981).

Quanto ao índice de erosividade $\mathrm{EI}_{30}$, a Estação Posto Garrafão apresentou erosividade das chuvas 283,8\% maior que a Estação Manuel Duarte. Quando esta comparação é feita por meio do índice KE > 25, tal percentual chega a $308,1 \%$.

Ao se analisar, entretanto, a série histórica de dados utilizada na obtenção dos índices de erosividade, verifica-se que o Posto Garrafão apresenta dados de 1980 a 1995 (16 anos) e Manuel Duarte apenas do período de 1989 a 1994 (5 anos). Em razão deste fato resolveu-se determinar, também, os índices de erosividade somente do período de dados comuns entre as duas estações, ou seja, de 1989 a 1994, com a obtenção de valores de $\mathrm{EI}_{30}$ e $\mathrm{KE}>25$, no Posto Garrafão, de 18.155 $\mathrm{MJ} \mathrm{mm} \mathrm{ha}^{-1} \mathrm{~h}^{-1}$ ano $^{-1}$ e $195,7 \mathrm{MJ} \mathrm{ha}^{-1} \mathrm{ano}^{-1}$ e de 4.118 MJ mm ha-1 $\mathrm{h}^{-1}$ ano $^{-1}$ e 42,1 $\mathrm{MJ} \mathrm{ha}^{-1} \mathrm{ano}^{-1}$ em Manuel Duarte, respectivamente.

Observa-se, portanto, que a diferença percentual entre os locais com maior e menor erosividades aumentou ainda mais, atingindo $340,9 \%$ quando se considerou o $\mathrm{EI}_{30}$ e $364,8 \%$ para o KE > 25. Esta grande variação de erosividade nos diferentes locais estudados pode ser explicada, em parte, pelo fator orográfico. Enquanto a Estação Manuel Duarte se encontra na região Centro-Sul do Estado do Rio de Janeiro, a do Posto Garrafão fica bem na crista da região Serrana. De acordo com estudo de chuvas intensas feito pela CPRM (2000), nenhuma outra serra do território nacional exerce tanta influência no aumento das precipitações quanto as serras do Mar e da Mantiqueira, localizadas naquele Estado.

Na Tabela 3 estão apresentados, em ordem decrescente de percentual de ocorrência, o número médio de chuvas por ano, o número médio de chuvas erosivas por ano e a percentagem de chuvas erosivas em relação ao total de chuvas, além da precipitação total anual, precipitação considerada erosiva e percentagem de precipitação erosiva com relação ao total anual de cada uma das 14 estações pluviográficas utilizadas no estudo.

Nesta tabela são apresentadas, também, as medidas estatísticas relacionadas ao número de chuvas e à precipitação.

Observando-se os dados da Tabela 3, nota-se que o coeficiente de variação do número de chuvas total anual médio apresentou valores menores (18\%) que o coeficiente de variação do número de chuvas erosivas anual médio (26\%); o mesmo vale para a precipitação, sendo o coeficiente de variação total (32,3\%) inferior ao das chuvas erosivas (37,1\%).

O maior número absoluto anual médio de chuvas erosivas foi o da localidade de Posto Garrafão, com 75 chuvas, correspondendo a 41,9\% do número anual médio total. Apesar disso, Posto Garrafão não foi a estação com o maior percentual relativo, pois a Estação de Vila Mambucaba, com $44,3 \%$, apresentou a maior percentagem de chuvas erosivas em relação ao número total de chuvas.

Tabela 3. Número médio de chuvas por ano, número médio de chuvas erosivas por ano, percentagem de chuvas erosivas em relação ao total de chuvas, precipitação total anual, precipitação considerada erosiva, percentagem de precipitação erosiva em relação ao total anual e as medidas estatísticas relacionadas ao número de chuvas e à precipitação

\begin{tabular}{|c|c|c|c|c|c|c|}
\hline \multirow{2}{*}{ Estação } & \multicolumn{3}{|c|}{ Número de Chuvas } & \multicolumn{3}{|c|}{ Precipitação Média } \\
\hline & Ano & Erosiva & $(\%)$ & Total (mm) & Erosiva (mm) & $(\%)$ \\
\hline Vila Mambucaba & 122 & 54 & 44,3 & $2.033,9$ & $1.764,0$ & 86,7 \\
\hline Posto Garrafão & 179 & 75 & 41,9 & $3.006,0$ & $2.478,3$ & 82,4 \\
\hline Tocos & 128 & 49 & 38,5 & $1.465,6$ & $1.356,8$ & 92,6 \\
\hline Santa Isabel do R. Preto & 124 & 47 & 38,1 & $1.550,1$ & $1.259,7$ & 81,3 \\
\hline Manuel Duarte & 93 & 35 & 38,0 & $1.027,9$ & 810,8 & 78,9 \\
\hline Ilha dos Pombos & 93 & 34 & 36,8 & $1.013,0$ & 877,5 & 86,6 \\
\hline Capela Mayrink & 143 & 52 & 36,6 & $2.170,3$ & $1.864,8$ & 85,9 \\
\hline Escola União & 147 & 51 & 34,8 & $1.859,5$ & $1.497,5$ & 80,5 \\
\hline Cachoeiras de Macacu & 158 & 53 & 33,5 & $1.914,9$ & $1.467,2$ & 76,6 \\
\hline Eletrobrás & 116 & 35 & 30,6 & $1.280,0$ & 964,4 & 75,3 \\
\hline Tanguá & 131 & 39 & 29,4 & $1.387,3$ & $1.015,3$ & 73,2 \\
\hline Rio Mole & 122 & 34 & 27,6 & $1.252,3$ & 953,8 & 76,2 \\
\hline Lajes & 149 & 40 & 27,1 & $1.450,7$ & $1.045,6$ & 72,1 \\
\hline Santa Cecília & 138 & 33 & 24,0 & $1.486,2$ & 825,1 & 55,5 \\
\hline Média & 132 & 45 & 34 & $1.635,5$ & $1.298,6$ & 78,8 \\
\hline D. P. & 23 & 12 & & 528,8 & 481,7 & \\
\hline C. V. (\%) & 18 & 26 & & 32,3 & 37,1 & \\
\hline E. P. M. & 6 & 3 & & 132,2 & 120,4 & \\
\hline
\end{tabular}


Posto Garrafão, estação com maior total anual médio precipitado (3.006,0 mm), possui um percentual relativo de precipitações erosivas de $82,4 \%$, percentual este inferior ao das Estações Tocos, Vila Mambucaba, Ilha dos Pombos e Capela Mayrink, cujos valores foram de 92,6; 86,7; 86,6 e 85,9\%, respectivamente.

Outra constatação foi de que a precipitação anual média erosiva da Estação Santa Cecília (825,1 mm), que apresentou o menor percentual de precipitações erosivas, foi três vezes menor que a da Estação Posto Garrafão, com 2.478,3 mm. Comparando-se as precipitações totais anuais médias, esta relação passa a ser duas vezes menor nessas estações, ou seja, 1.486,2 e 3.006,0 mm, respectivamente.

Na Tabela 4 são apresentadas as equações de regressão ajustadas para a estimativa dos índices de erosividade $\mathrm{EI}_{30}$ e $\mathrm{KE}>25$ a partir de dados pluviométricos. Todas as equações foram significativas em nível de $1 \%$ de probabilidade, pelo teste $\mathrm{F}$, e os coeficientes de determinação $\left(\mathrm{r}^{2}\right)$ variaram de 0,61 a 0,99. A exceção foi a Estação Capela Mayrink, que apresentou coeficientes de determinação em torno de 0,40.

Quando se usou o coeficiente de chuva (Rc) ao invés da precipitação mensal média (p) para estimar a erosividade, não houve aumento significativo no valor do coeficiente de determinação; portanto, desejando estimar a erosividade das chuvas nas regiões estudadas, pode-se simplesmente empregar a precipitação mensal média. Devido aos maiores coeficientes de determinação encontrados, é possível dizer que o $\mathrm{EI}_{30}$ poderá ser estimado com mais segurança que o KE > 25.

Na Figura 2 (A e B) estão apresentados os mapas temáticos com os índices de erosividades anuais médios $\mathrm{EI}_{30}$ e KE $>25$ do Estado do Rio de Janeiro. Ressalta-se, entretanto, que nesses mapas não estão contempladas as regiões Norte e Noroeste do Estado, por falta de estações pluviográficas.

Os mapas foram gerados com classes de $1.000 \mathrm{MJ} \mathrm{mm} \mathrm{ha-1} \mathrm{h}^{-1} \mathrm{ano}^{-1}$ para o índice de erosividade $\mathrm{EI}_{30}$ e $10 \mathrm{MJ} \mathrm{ha}^{-1} \mathrm{ano}^{-1}$ para $\mathrm{KE}>25$. Para o índice $\mathrm{EI}_{30}$
A.

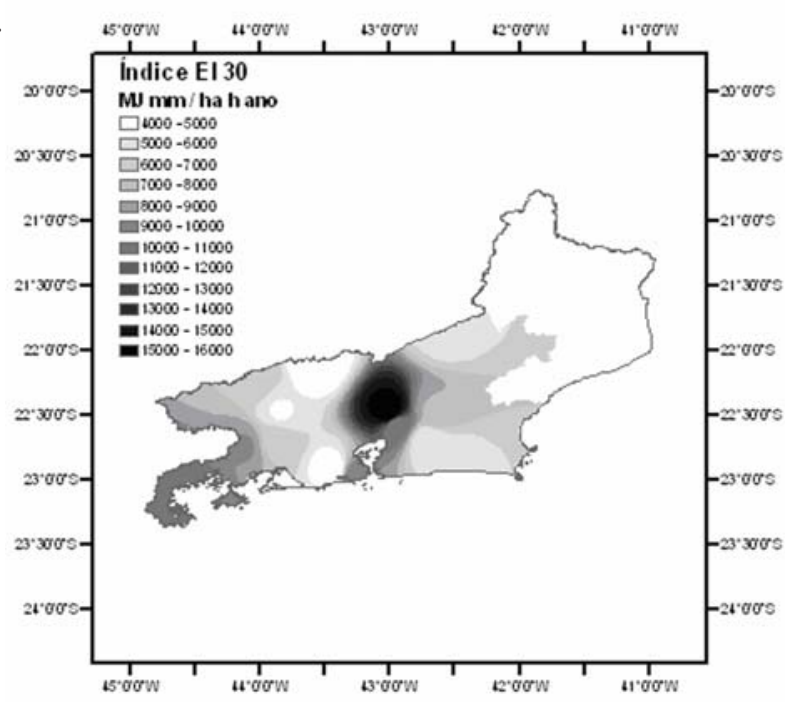

B.

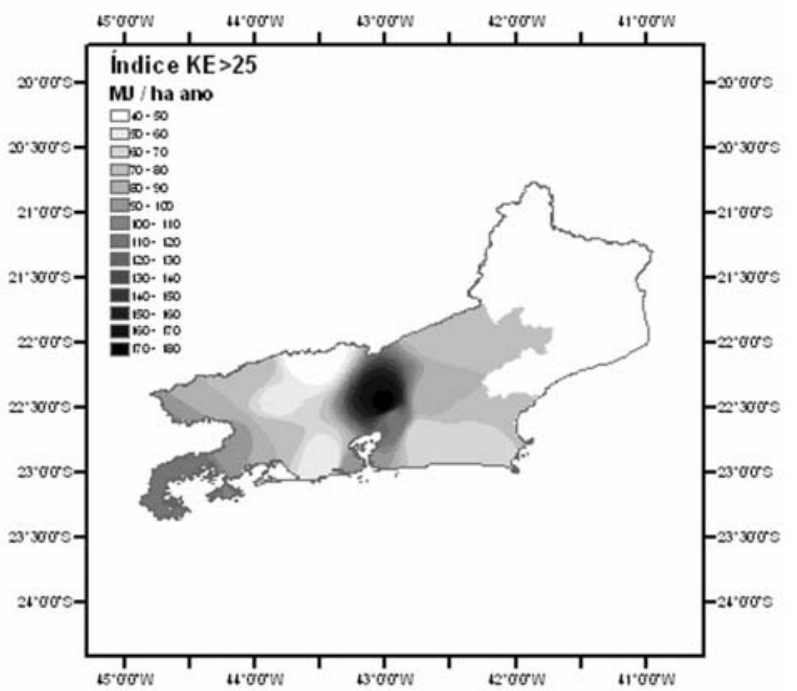

Figura 2. Mapas de isoerosividade do Estado do Rio de Janeiro, obtido por meio de interpolação do índice de erosividade $\mathrm{EI}_{30}(\mathrm{~A})$ e, obtido por meio de interpolação do índice de erosividade KE > 25 (B)

Tabela 4. Equações de regressão ajustadas entre os índices de erosividade mensal médio $E l_{30}\left(M J \mathrm{~mm} \mathrm{ha}^{-1} \mathrm{~h}^{-1}\right.$ ano-1) e KE $>25\left(M J \mathrm{ha}^{-1} \mathrm{ano}^{-1}\right)$ e a precipitação média mensal (P) ou o coeficiente de chuva (Rc)

\begin{tabular}{|c|c|c|c|c|c|c|c|c|}
\hline \multirow{2}{*}{ Estação } & \multicolumn{4}{|c|}{ Índice $\mathrm{El}_{30}$} & \multicolumn{4}{|c|}{ Índice KE > 25} \\
\hline & $P(\mathrm{~mm})$ & $\mathrm{r}^{2}$ & $\mathrm{Rc}(\mathrm{mm})$ & $\mathrm{r}^{2}$ & $P(\mathrm{~mm})$ & $\mathrm{r}^{2}$ & $\mathrm{Rc}(\mathrm{mm})$ & $r^{2}$ \\
\hline CM & $\mathrm{El} 30=6,94 \mathrm{p}-444,62$ & 0,92 & $\mathrm{El} 30=37,90 \mathrm{Rc}+39,86$ & 0,91 & $\mathrm{KE}>25=0,09 \mathrm{p}-5,83$ & 0,88 & $\mathrm{KE}>25=0,46 \mathrm{Rc}+0,23$ & 0,86 \\
\hline CMA & El30 $=9,49$ p- 938,28 & 0,41 & $\mathrm{El} 30=53,31 \mathrm{Rc}-76,27$ & 0,40 & $\mathrm{KE}>25=0,11 p-12,89$ & 0,39 & $\mathrm{KE}>25=0,63 \mathrm{Rc}-3,01$ & 0,38 \\
\hline$E$ & El30 $=6,28 p-300,13$ & 0,77 & $\mathrm{E} \mid 30=37,76 \mathrm{Rc}+3,89$ & 0,79 & $K E>25=0,09 p-4,87$ & 0,76 & $\mathrm{KE}>25=0,52 \mathrm{Rc}-0,75$ & 0,79 \\
\hline EU & El30 $=8,97 p-537,38$ & 0,91 & $\mathrm{El} 30=47,68 \mathrm{Rc}+64,59$ & 0,89 & $\mathrm{KE}>25=0,11 \mathrm{p}-7,15$ & 0,90 & $\mathrm{KE}>25=0,59 \mathrm{R} c+0,25$ & 0,88 \\
\hline IP & $\mathrm{El} 30=5,70 \mathrm{p}-9,74$ & 0,86 & $\mathrm{El} 30=21,00 \mathrm{Rc}+223,87$ & 0,72 & $\mathrm{KE}>25=0,07 p+0,12$ & 0,82 & $\mathrm{KE}>25=0,26 \mathrm{Rc}+3,01$ & 0,68 \\
\hline $\mathrm{L}$ & $\mathrm{El} 30=4,62 \mathrm{p}-143,58$ & 0,88 & $\mathrm{El} 30=22,69 \mathrm{Rc}+95,40$ & 0,82 & $K E>25=0,06 p-2,01$ & 0,88 & $\mathrm{KE}>25=0,29 \mathrm{R} c+1,04$ & 0,83 \\
\hline MD & $\mathrm{El} 30=4,37 \mathrm{p}-42,90$ & 0,89 & $\mathrm{El} 30=20,70 \mathrm{Rc}+112,54$ & 0,82 & $K E>25=0,04 p-0,10$ & 0,72 & $\mathrm{KE}>25=0,18 \mathrm{Rc}+1,36$ & 0,62 \\
\hline$P G$ & El30 $=8,01 p-689,41$ & 0,72 & $\mathrm{El} 30=46,37 \mathrm{Rc}+146,28$ & 0,70 & $K E>25=0,10 p-9,95$ & 0,69 & $\mathrm{KE}>25=0,57 \mathrm{Rc}-0,03$ & 0,69 \\
\hline $\mathrm{RM}$ & El30 $=8,29 p-411,50$ & 0,65 & $\mathrm{El} 30=50,02 \mathrm{Rc}-13,36$ & 0,65 & $K E>25=0,10 p-5,02$ & 0,61 & $\mathrm{KE}>25=0,59 \mathrm{Rc}-0,34$ & 0,61 \\
\hline SC & $\mathrm{El} 30=4,44 \mathrm{p}-134,29$ & 0,94 & $\mathrm{El} 30=24,53 \mathrm{Rc}+50,36$ & 0,96 & $K E>25=0,06 p-1,82$ & 0,92 & $\mathrm{KE}>25=0,31 \mathrm{Rc}+0,52$ & 0,94 \\
\hline SI & El30 = 4,82 p-24,52 & 0,90 & $\mathrm{El} 30=27,74 \mathrm{Rc}+194,08$ & 0,82 & $K E>25=0,06 p-0,66$ & 0,87 & $\mathrm{KE}>25=0,33 \mathrm{Rc}+2,19$ & 0,83 \\
\hline TA & El30 $=6,21 p-277,44$ & 0,85 & $\mathrm{El} 30=35,13 \mathrm{Rc}+38,48$ & 0,81 & $K E>25=0,08 p-4,06$ & 0,80 & $\mathrm{KE}>25=0,46 \mathrm{Rc}-0,03$ & 0,77 \\
\hline T0 & El30 $=8,24$ p-256,93 & 0,99 & $\mathrm{El} 30=38,48 \mathrm{Rc}+118,71$ & 0,98 & $K E>25=0,11 p-3,53$ & 0,97 & $\mathrm{KE}>25=0,50 \mathrm{R} c+1,34$ & 0,97 \\
\hline VM & $\mathrm{El} 30=8,50 \mathrm{p}-549,22$ & 0,81 & $\mathrm{El} 30=44,61 \mathrm{Rc}+73,21$ & 0,84 & $\mathrm{KE}>25=0,08 p-5,93$ & 0,70 & $\mathrm{KE}>25=0,44 \mathrm{Rc}+0,02$ & 0,74 \\
\hline
\end{tabular}

CM - Capela Maryrink, CMA - Capela Mayrink, E - Eletrobrás, EU - Escola União, PI- Ilha dos Pombos, L- Lages, MD - Manuel Duarte, PG - Posto Garrafão, RM - Rio Mole, SC - Santa Cecília, SI - Santa Izabel do Rio Preto, TA - Tangua, TO - Tocos, VM - Vila Mambucaba 
foram encontradas 12 classes de índices de erosividade variando de 4.000 a $16.000 \mathrm{MJ} \mathrm{mm} \mathrm{ha}^{-1} \mathrm{~h}^{-1} \mathrm{ano}^{-1} \mathrm{e}$, para o índice KE $>25$, obtiveram-se 14 classes de índices de erosividade oscilando de 40 a $180 \mathrm{MJ} \mathrm{ha}^{-1} \mathrm{ano}^{-1}$.

Para o índice $\mathrm{EI}_{30}$, as classes de erosividade possuem valores da ordem de 10.000 a $11.000 \mathrm{MJ} \mathrm{mm} \mathrm{ha}^{-1} \mathrm{~h}^{-1} \mathrm{ano}^{-1}$ na parte mais litorânea da região Sul, chegando a valores máximos, próximos a $16.000 \mathrm{MJ} \mathrm{mm} \mathrm{ha}^{-1} \mathrm{~h}^{-1} \mathrm{ano}^{-1}$, na região Serrana e decrescendo em direção ao norte, com valores da ordem de $6.000 \mathrm{MJ} \mathrm{mm} \mathrm{ha}^{-1} \mathrm{~h}^{-1} \mathrm{ano}^{-1}$. A região Centro-Sul apresentou os menores valores de $\mathrm{EI}_{30}$, ao redor de 4.000 MJ mm ha-1 $\mathrm{h}^{-1} \mathrm{ano}^{-1}$. O índice $\mathrm{KE}>25$ teve o mesmo comportamento do índice $\mathrm{EI}_{30}$.

Algumas considerações devem ser feitas para a utilização desses mapas, pois eles servem apenas como uma primeira aproximação necessitando, portanto, do fornecimento de mais dados para que possam ser gerados resultados mais confiáveis. Uma das opções para a melhoria das informações seria uma interpolação que correlacionasse a altitude local, o índice de erosividade e a precipitação média. O Estado do Rio de Janeiro tem peculiaridades específicas, como a já mencionada presença das Serras do Mar e da Mantiqueira, que fazem com que ocorram altos índices pluviométricos na região, contrastando com regiões de baixo índice de chuvas, como o Vale do Paraíba; por isso, mais localidades devem ser analisadas para complementar as informações obtidas por meio deste trabalho.

\section{CONCLUSÕES}

1. Os índices de erosividade das chuvas apresentam, para os diferentes locais estudados, diferenças expressivas entre si, sendo a região Serrana aquela que apresenta os maiores valores de erosividade, e a região Centro-Sul a que mostra os menores valores.

2. Não houve diferença expressiva entre as equações propostas por Foster et al. (1981) e Wagner \& Massambani (1988) para o cálculo da energia cinética das chuvas.

3. A estimativa da erosividade das chuvas a partir da precipitação média mensal (p) proporcionou melhores resultados que quando se usou o coeficiente de chuva (Rc).

4. O número médio anual de chuvas erosivas nas localidades analisadas foi de 45, correspondendo a 34\% do número médio anual total de eventos.

\section{AgRADECIMENTOS}

À Agência Nacional de Águas/Agência Nacional de Energia Elétrica (ANA/ANEEL) à Light Serviços de Eletricidade S/A (Light) e à Superintendência Estadual de Rios e Lagoas (SERLA), pelo fornecimento dos dados pluviográficos.

\section{LITERATURA CITADA}

Cabeda, M. S. V. Computation of storm EI value. West Lafayette: Purdue University, 1976. 6p.

Chaves, I. B.; Diniz, E. J. Erosividade das chuvas no Estado da Paraíba. In: Encontro Nacional de Pesquisa sobre Conservação do Solo, 3, 1981, Recife. Resumos... Recife: SBCS, 1981. p.136-147.

CPRM - Companhia de Pesquisa de Recursos Minerais. Estudo de chuvas intensas no estado do Rio de Janeiro. Belo Horizonte: CPRM, 2000. 139p.

Foster, G. R.; Mccool, D. K.; Renard, K. G.; Moldenhauer, W. C. Conversion of the universal soil loss equation to SI units. Journal of Soil and Water Conservation, Baltimore, v.36, p.355-359, 1981.

Hudson, N. W. Soil conservation. Ithaca: Cornell University Press, 1973. 320p.

Lago, J. C. Erosividade das chuvas de Pelotas. In: Encontro Nacional de Pesquisa sobre Conservação do Solo, 5, 1984, Porto Alegre. Resumos... Porto Alegre: SBCS, 1984. p.72.

Lal, R. Erodibility and erosivity. In: Lal, R. Soil erosion research methods. Ankeny: SWCS, 1988. p.141-160.

Lombardi Neto, F. Rainfall erosivity - Its distribution and relationship with soil loss at Campinas, Brazil. West Lafayette: Purdue University, 1977. 53p. M.Sc. Dissertation

Marques, J. J. G. S. M.; Alvarenga, R. C.; Curi, N. Erosividade das chuvas da região de Sete Lagoas, MG. Pesquisa Agropecuária Brasileira, Brasília, v.33, n.5, p.285-288, 1988.

Marques, J. J. G. S. M.; Alvarenga, R. C.; Curi, N.; Santana, D. P.; Silva, M. L. N. Índices de erosividade da chuva, perdas de solo e fator erodibilidade para dois solos da região dos cerrados - primeira aproximação. Revista Brasileira de Ciência do Solo, Campinas, v.21, n.3, p.427-434, 1997.

McGregor, K. C.; Binger, R. L.; Bowie, A. J.; Foster, G. R. Erosivity index values for northern Mississippi. Transactions of the ASAE, St. Joseph, v.38, n.4, p.1039-1047, 1995.

Medina, B. F.; Oliveira Júnior, R. C. A aplicabilidade de alguns índices erosivos em Latossolo Amarelo de Manaus, AM. Revista Brasileira de Ciência do Solo, Campinas, v.11, n.1, p.67-70, 1987.

Morais, L. F. B.; Silva, V.; Naschenveng, T. M. C.; Hardoin, P. C.; Almeida, J. E. L.; Weber, O. L. S.; Boel, E.; Durigon, V. Índice $\mathrm{EI}_{30}$ e sua relação com o coeficiente de chuva do sudoeste do Mato Grosso. Revista Brasileira de Ciência do Solo, Campinas, v.15, n.3, p.339-344, 1991.

Moura, A. R. B.; Medeiros, J. F. Determinação inicial da erosividade da chuva (fator R) em 1985, em Mossoró, RN. Revista Brasileira de Ciência do Solo, Campinas, v.11, n.2, p.229231, 1987.

Oliveira Júnior, R. C. A erosividade das chuvas na parte leste do Estado do Pará. Belém: FCAP, 1988. 52p. Dissertação Mestrado

Oliveira Júnior, R. C.; Medina, B. F. A erosividade das chuvas em Manaus, AM. Revista Brasileira de Ciência do Solo, Campinas, v.14, n.2, p.235-9, 1990.

Rufino, R. L. Avaliação do potencial erosivo da chuva para o Estado do Paraná: segunda aproximação. Revista Brasileira de Ciência do Solo, Campinas, v.10, n.3, p.279-81, 1986. 
Salako, F. K.; Ghuman, B. S.; Lal, R. Rainfall erosivity in south-central Nigeria. Soil Technology, New York, v.7, n.4, p.279-90, 1995.

Silva, M. L. N.; Freitas, P. L.; Blancaneaux, P.; Curi, N. Índices de erosividades das chuvas da região de Goiânia, GO. Pesquisa Agropecuária Brasileira, Brasília, v.32, n.10, p.275289. 1997.

Wagner, C. S.; Massambini, O. Análise da relação intensidade de chuva: energia de Wischmeier \& Smith e sua aplicabilidade à região de São Paulo. Revista Brasileira de Ciência do Solo, Campinas, v.12, n.3, p.197-203, 1988.
Wischmeier, W. H.; Smith, D. D. Rainfall energy and its relationship to soil loss. Transaction American Geophysical Union, Washington, v.39, n.2, p.285-91, 1958.

Wischmeier, W. H.; Smith, D. D. Predicting rainfall erosion losses: a guide to conservation planning. Washington, DC: USDA, 1978. 58p. Agriculture Handbook 537

Wischmeier, W. H.; Smith, D. D. Predicting rainfall-erosion losses from cropland east of the Rocky Mountains: Guide for selection of practices for soil and water conservation. Washington, DC: USDA, 1965. 47p. Agricultural Handbook 282 